\title{
Bed-Load Effects on Hydrodynamics of Rough-Bed Open-Channel Flows
}

\author{
Lorna Campbell ${ }^{1}$; Ian McEwan²; Vladimir Nikora³; Dubravka Pokrajac ${ }^{4}$; Michael Gallagher; and \\ Costantino Manes ${ }^{6}$
}

\begin{abstract}
The extent to which turbulent structure is affected by bed-load transport is investigated experimentally using a nonporous fixed planar bed comprising mixed-sized granular sediment with a $d_{50}$ of $1.95 \mathrm{~mm}$. Three different sizes of sediment $\left(d_{50}=0.77,1.99\right.$, and $3.96 \mathrm{~mm}$ ) were fed into the flow at two different rates $(0.003$ and $0.006 \mathrm{~kg} / \mathrm{m} / \mathrm{s})$, and subsequently transported as bed load. Particle image velocimetry (PIV) was used to determine the turbulence characteristics over the fixed bed during clear water and sediment feed cases. Mean longitudinal flow velocities at any given depth were lower than their clear water counterparts for all but one of the mobile sediment cases. The exception was with the transport of fine grains at the higher feed rate. In this case, longitudinal mean flow velocities increased compared to the clear water condition. The coarse grains tended to augment bed roughness, but fine grains saturated the troughs and interstices in the bed topography, effectively causing the influence of bed irregularities to be smoothed. The PIV technique permitted examination of both temporal and spatial fluctuations in flow variables: therefore many results are presented in terms of double-averaged quantities (in temporal and spatial domains). In particular, the form-induced stress, which arises from spatially averaging the Reynolds averaged Navier-Stokes equations and is analogous to the Reynolds turbulent stress, contributed between 15 and $35 \%$ of the total measured shear stress in the roughness layer. Flow around protrusive roughness elements produced a significant proportion of the turbulent kinetic energy shear production, suggesting that this process is highly intermittent near rough beds.
\end{abstract}

DOI: 10.1061/(ASCE)0733-9429(2005)131:7(576)

CE Database subject headings: Sediment transport; Bed loads; Turbulence; Open channel flow; Hydrodynamics; Channel beds.

\section{Introduction}

The majority of natural and man-made open-channel flows (e.g., river channels and canals) are fully developed turbulent flows over rough mobile beds. Mean velocity distribution and turbulence structure above the bed roughness in open channels is fairly well understood for clear water flows in the absence of sediment transport (Nezu and Nakagawa 1993). However, there is a call for engineers to develop a deeper understanding of the complex feedback mechanisms between sediment transport and flow hydrody-

\footnotetext{
${ }^{1}$ Research Assistant, Dept. of Engineering, Univ. of Aberdeen, Fraser Noble Building, Aberdeen AB24 3UE, U.K. E-mail: 1.j.campbell@ eng.abdn.ac.uk

${ }^{2}$ Reader, Dept. of Engineering, Univ. of Aberdeen, Fraser Noble Building, Aberdeen AB24 3UE, U.K. E-mail: i.mcewan@eng.abdn.ac.uk

${ }^{3}$ Principal Scientist, Freshwater Hydrodynamics, National Institute of Water and Atmospheric Research, P.O. Box 8602, Christchurch, New Zealand. E-mail: v.nikora@niwa.co.nz

${ }^{4}$ Lecturer, Dept. of Engineering, Univ. of Aberdeen, Fraser Noble Building, Aberdeen AB24 3UE, U.K. E-mail: d.pokrajac@eng.abdn.ac.uk

${ }^{5}$ Dept. of Engineering, Univ. of Aberdeen, Aberdeen AB24 3UE, U.K.; formerly, Research Assistant

${ }^{6}$ Postgraduate Student, Dept. of Engineering, Univ. of Aberdeen, Fraser Noble Building, Aberdeen AB24 3UE, U.K. E-mail: c.manes@ eng.abdn.ac.uk

Note. Discussion open until December 1, 2005. Separate discussions must be submitted for individual papers. To extend the closing date by one month, a written request must be filed with the ASCE Managing Editor. The manuscript for this paper was submitted for review and possible publication on December 11, 2002; approved on November 2, 2004. This paper is part of the Journal of Hydraulic Engineering, Vol. 131, No. 7, July 1, 2005. CASCE, ISSN 0733-9429/2005/7-576-585/\$25.00.
}

namics in alluvial rivers. Although many studies have focussed on the effect of mean flow properties on sediment transport, relatively few have considered the converse, i.e., the influence such transport exerts on the mean and turbulent flow properties. The impact of sediment transport on mean velocity gradients, turbulence intensities, Reynolds stress and turbulent kinetic energy (TKE) generation is of much interest.

Investigations into the feedback effect of sediment transport on flow hydrodynamics have tended to focus on sediment transported in suspension (e.g., Vanoni and Nomicos 1960; Wang and Ning 1989), or material carried both in suspension and as bedload (e.g., Muller 1973). Relatively few studies have concentrated purely on bed-load transport, but there is uncertainty regarding the effect of sediment transport on the mean flow and turbulence characteristics regardless of transport mode. Vanoni and Nomicos (1960) concluded that suspended sediment damped turbulence intensity and hence reduced flow resistance, whereas Müller (1973) found the opposite, with increased streamwise turbulence intensity in the presence of mobile sediment. This apparent contradiction is echoed in bed-load studies.

In general, flow resistance increases with bed-load transport compared to clear water conditions (Gust and Southard 1983; Wang and Larsen 1994; Best et al. 1997; Song and Chiew 1997). Coarse sand (Wang and Larsen 1994) and $0.22 \mathrm{~mm}$ diameter glass spheres (Best et al. 1997) carried as bed load resulted in lower longitudinal velocities, and increased velocity gradients and turbulence intensities in the near-bed region. This is consistent with the view that bed-load collisions extract kinetic energy from the mean flow leading to a near-bed momentum deficit, and hence reduced streamwise velocity (Owen 1964; Smith and McLean 1977). Bed-load grains interact not only with the flow, but also 
with the bed. The flow acts to accelerate the grains, whereas the bed decelerates them until they ultimately come to rest (Gyr and Schmid 1997). However, recent work suggests that bed load can instead reduce flow resistance, resulting in increased downstream velocity. For example, Nikora and Goring (2000) found that weak gravel bed load in a New Zealand irrigation canal increased mean longitudinal flow velocities. Pitlick (1992) reported that developing bed forms in the North Fork Toutle River, Wash. greatly increased flow resistance, but weak bed load over a planar gravel bed had little effect when compared to clear water flows. Flow resistance may therefore be increased, decreased, or unchanged by bed-load transport.

Recent studies [e.g., Gore and Crowe 1989 (suspended sediment); Carbonneau and Bergeron 2000 (bed load)] have attempted to clarify the apparent contradiction regarding flow resistance in sediment transporting flows. Carbonneau and Bergeron (2000) used a well-sorted gravel $\left(d_{50}=7.4 \mathrm{~mm}\right)$ for their fixed bed and bed-load sediment, and conducted four smooth bed and three rough bed experiments with varying transport rate. For two of three rough bed runs, bed-load transport caused an increase rather than a decrease of flow velocity, demonstrating that the effect of such sediment transport on mean flow characteristics is complex.

Regardless of whether bed-load transport increases, decreases, or has a negligible effect on flow resistance, it is clear that mean and turbulent flow properties over a mobile bed may differ significantly from those over a fixed bed. However, the available information on potential differences is still limited and relates to fairly narrow ranges of control parameters and/or experimental scenarios. For example Best et al. (1997) used uniform glass spheres for both the fixed bed and bed-load sediment such that the effects of fine sediment filling the fixed bed pore space could not be addressed. It is possible that the response of the flow would be altered if the rolling bed-load grains could be freely exchanged with troughs and pores in the bottom sediment. Thus the decision to use mixed grain sized sediments as bed load in this study provides an increased level of complexity over some previous studies. In particular, the influence of varying bed-load sediment size distributions and transport rates on turbulence characteristics and mean velocities requires clarification.

In this paper we examine the effects on turbulent flow structure of three different sizes of feed material $\left(d_{50}=0.77,1.99\right.$, and $3.96 \mathrm{~mm}$ ) transported as bed load over a nonporous fixed planar bed $\left(d_{50}=1.95 \mathrm{~mm}\right)$. Furthermore, data collection by means of a particle image velocimetry (PIV) system has enabled quantification and visualization of the spatial variability in the timeaveraged flow field, such as form-induced stresses and the occurrence of localized TKE production "hot spots."

\section{Experimental Conditions}

The experiments were carried out in a recirculating hydraulic flume $12.5 \mathrm{~m}$ long and $0.3 \mathrm{~m}$ wide. The flow depth varied between 64 and $68 \mathrm{~mm}$, resulting in a width-to-depth ratio $(B / H)$ of approximately 4.5. $\mathrm{R}=\bar{U} R / v$ was on the order of 25,000 and the Froude number $\mathrm{F}=\bar{U} / \sqrt{ }(\mathrm{g} R)$ varied from 0.82 to 0.84 , where $\bar{U}$ is the cross-sectional mean flow velocity, $R$ is the hydraulic radius, $v$ is kinematic viscosity, and $g$ is gravity acceleration. For all experiments, the slope of the flume was set to $1 / 250$ and a constant discharge of $11 \mathrm{~L} / \mathrm{s}$ was set. Uniform flow was maintained throughout. A sediment mixture (with a $d_{50}$ value of $1.95 \mathrm{~mm}$ - see Fig. 1) was glued to the smooth wooden base of

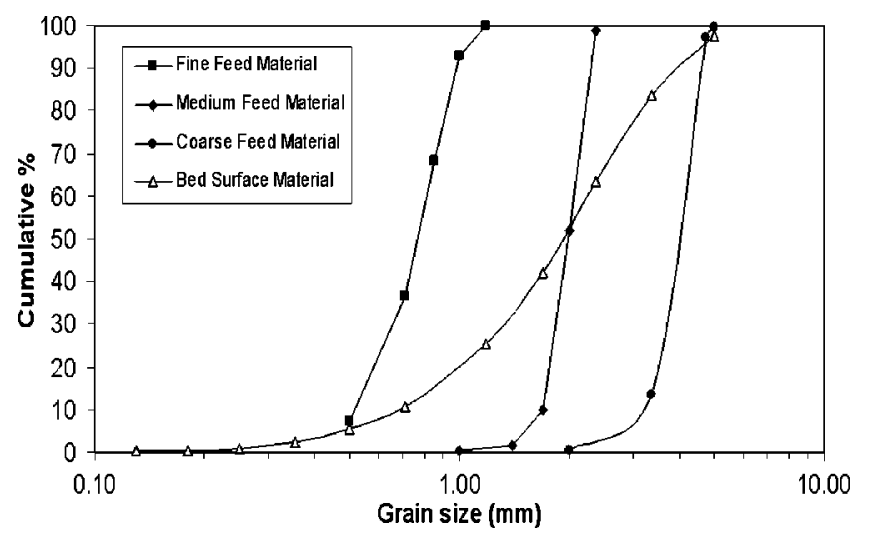

Fig. 1. Grain size distributions (by weight) of fixed bed surface and bed-load feeding sediment. Fixed bed $d_{50}=1.95 \mathrm{~mm}$, fine, medium, and coarse bed-load $d_{50}=0.77,1.99$, and $3.96 \mathrm{~mm}$, respectively.

the flume to provide a constant uniform bed roughness. All measurements were conducted for hydraulically rough bed conditions, with the roughness Reynolds number $\mathrm{R}_{*}=u_{*} d_{50} / v>100$, where $u_{*}$ is friction velocity.

In each experiment the flow structure over the fixed bed with no sediment transport was measured ("clear water"), and then the flow structure was measured during the bed-load transport of fine $\left(d_{50}=0.77 \mathrm{~mm}\right)$, medium $\left(d_{50}=1.99 \mathrm{~mm}\right)$, and coarse $\left(d_{50}\right.$ $=3.96 \mathrm{~mm})$ grains fed at two different rates $(0.003$ and $0.006 \mathrm{~kg} / \mathrm{m} / \mathrm{s}$ ). The size distributions of the fed sediment are shown in Fig. 1. The feeding sediment was transported purely as bed load with grains rolling rather than saltating. However, as the size of the feeding sediment increased the grains tended to become temporarily lodged on the fixed bed. Therefore, to ensure that the initial fixed bed roughness had not changed across all trials, the bed surface was brushed before commencing each experiment. The sediment was fed into the flow at a constant feed rate via a hopper attached to a gearing mechanism placed $3.34 \mathrm{~m}$ upstream of the flow measurement area. The transport capacity of the flow always exceeded the bed-load feed rate, such that the bed-load transport rate was equal to the feed rate. Thus, all mobile sediment was transported right through the whole length of the flume. No bedforms were developed. Changes to turbulent structure due to both the size and rate of the transported sediment in the absence of bedforms could therefore be examined. Table 1 provides information on the bed shear stresses, shear velocities, and Shields parameter for all experiments.

\section{Measurement and Analysis Techniques}

\section{Particle Image Velocimetry}

PIV was used to record flow velocities as it offers a number of advantages, for example fluid velocities over the entire flow depth can be measured synchronously in a nonintrusive manner. Additionally it is possible to calculate the mean bed level within the measurement window directly from the recorded PIV images (by taking the average elevation of the bed topography). PIV relies on stroboscopic illumination of a plane area of the flow which has been seeded with small neutrally buoyant particles. Conifer pollen with a mean diameter of $50 \mu \mathrm{m}$ was used as the seeding material. Multiply exposed PIV images were obtained from a vertical plane in the midline of the flume by repeatedly traversing the flow 
Table 1. Summary of Parameters Obtained from Shear Stress Profiles

\begin{tabular}{lccc}
\hline & Clear water & $\begin{array}{c}\text { Fine sediment } \\
\text { feed rate 1,2 }\end{array}$ & $\begin{array}{c}\text { Medium sediment } \\
\text { feed rate } 1,2\end{array}$ \\
\hline Parameters & 2.73 & $2.75,2.53$ & $\begin{array}{c}\text { Coarse sediment } \\
\text { feed rate } 1,2\end{array}$ \\
\hline Bed shear stress $\tau_{0}\left(\mathrm{~N} / \mathrm{m}^{2}\right)^{\mathrm{a}}$ & 0.054 & $0.054,0.052$ & $2.67,2.64$ \\
Shear velocity $u_{*}(\mathrm{~m} / \mathrm{s})^{\mathrm{b}}$ & - & $0.22,0.20$ & $0.053,0.054$ \\
Shields parameter $\theta^{\mathrm{c}}$ & - & $0.08,0.08$ & $0.054,0.058$ \\
\hline
\end{tabular}

Note: Rate $1=0.003 \mathrm{~kg} / \mathrm{m} / \mathrm{s}$ and rate $2=0.006 \mathrm{~kg} / \mathrm{m} / \mathrm{s}$.

${ }^{\mathrm{a}}$ Derived from linear extrapolation of the Reynolds stress profiles to the mean bed level.

${ }^{\mathrm{b}} u_{*}=\left(\tau_{0} / \rho\right)^{0.5}$.

${ }^{\mathrm{c} C a l c u l a t e d}$ from $\rho u_{*}^{2} /\left(\rho_{s}-\rho\right) \mathrm{g} d_{50}$.

measurement area with a scanning argon-ion laser beam [Fig. 2(a)]. As the polygonal mirror rotates, each face catches the laser beam and reflects the light to scan across the parabolic mirror. This in turn reflects the scanning beam vertically downwards through the seeded flume flow, allowing individual pollen particles to be illuminated. The scanning beam passed through the entire recording area every $1.8 \mathrm{~ms}$. Individual particles were therefore illuminated for a brief instant each time the scanning beam passed. In all experiments the laser beam scanned a flow area in the center line of the flume, and video images were taken in the vertical $(x, z)$ plane for a flow area of $67 \mathrm{~mm}$ (streamwise) $\times 61 \mathrm{~mm}$ (vertical). The images were obtained $8.34 \mathrm{~m}$ downstream of the flume inlet. A digital camera with a resolution of 1,000 by 1,000 pixels and an exposure time of $32 \mathrm{~ms}$ was used to record the flow field at a frequency of $16.7 \pm 0.2 \mathrm{~Hz}$. The selected exposure time of the camera enabled individual seeding particles to be recorded between 10 and 17 times in each image, thus providing a displacement record for the particles recorded in the measurement plane as shown in Fig. 2(b). Each experiment involved recording for $250 \mathrm{~s}$.

The software package VISIFLOW (AEA Technology, Oxfordshire, United Kingdom) was used for PIV analysis, which involved splitting each of the digitized frames into a grid of interrogation areas. The interrogation areas overlapped each other by $75 \%$. Each interrogation area corresponded to a physical flow area of $4.29 \mathrm{~mm}$ by $4.29 \mathrm{~mm}$, and was analyzed using the autocorrelation method (Shand 1996) to establish the average displacement of the particles within the area. Division of the displacement value by the time interval between each particle illumination resulted in a velocity magnitude. This magnitude plus the displacement orientation gives a flow velocity vector in the vertical plane, which was position-7ed at the center of the interrogation area. The analysis routine for each image produced 3,481 instantaneous velocity vectors.

The PIV vector maps contained a number of erroneous vectors because the autocorrelation routine had computed the average displacement incorrectly. Erroneous vectors were generated if there was insufficient seeding in a particular interrogation area, or if large displacements moved the pollen particles out of the interrogation area before subsequent illuminations. Due to the large number of frames being analyzed it was necessary to automate a velocity vector validation procedure. The chosen method was based on the work of Westerweel (1994), who classified vectors as erroneous if they varied significantly from their nearest neighbors. In this investigation, eight neighboring vectors were averaged and vectors varying by more than 2 standard deviations from the average value were defined as erroneous and deleted. The majority of erroneous vectors were concentrated near the bed where the interrogation areas began to overlap with the bed material. Also, the PIV autocorrelation algorithm cannot resolve negative (upstream) velocities where flow separates in the wake of individual grains. Therefore no data are presented for the region below the roughness tops. Furthermore, flow data were potentially compromised as bed-load sediment intermittent passed through the video field. Hence the authors propose a cautionary approach to data interpretation for the bottom $5 \%$ of the flow immediately above the fixed bed in the bed-load transport cases. Other inaccuracies associated with PIV analysis are: pixelation of the digital images that results in small errors in locating the correlation peak used to determine particle displacement, and hence velocity, and averaging particle velocities across an interrogation area, which is most sensitive in areas near the bed with large (a)

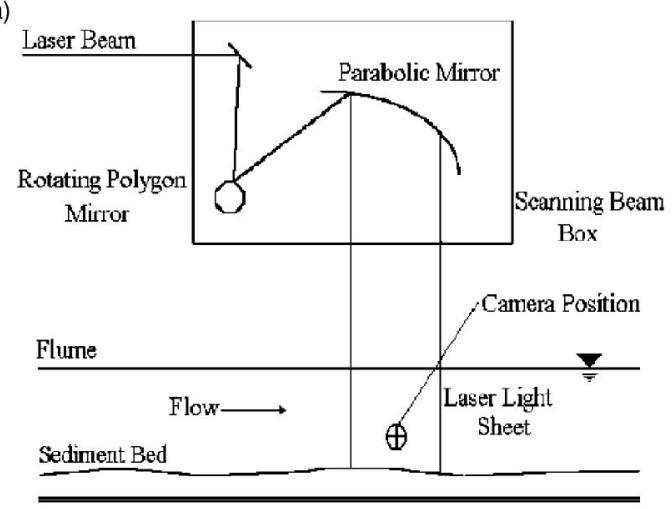

(b)

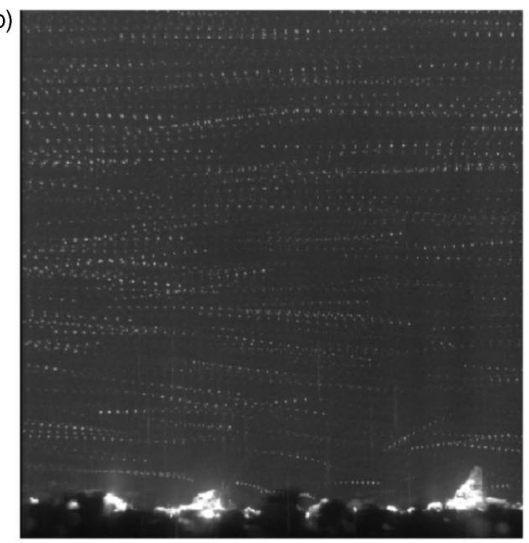

Fig. 2. (a) Scanning box system used to produce illumination for particle image velocimetry images; (b) autocorrelation particle image velocimetry frame from clear water case (flow area $67 \times 61 \mathrm{~mm}$, flow is from left to right) 


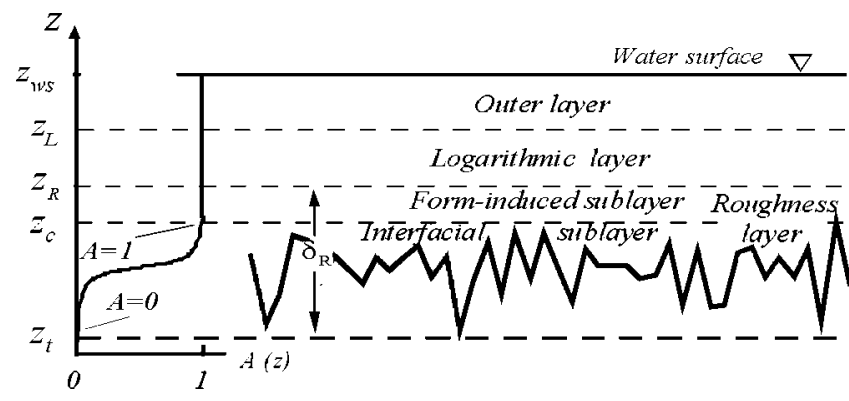

Fig. 3. Flow subdivision into specific regions for impermeable bed (adapted from Nikora et al. 2001)

velocity gradients. However, above the bottom $3 \%$ of the flow field, these errors are of the same order as those due to pixelation. The total error in velocity measurements varied from 0.5 to $4 \%$.

\section{Spatially-Averaged Momentum Equations}

Smith and McLean (1977) used spatial flow averaging to describe velocity profiles above a wavy bed. Subsequently the approach was developed by atmospheric physicists to provide a tool for assessing flow variables within plant canopies (Wilson and Shaw 1977; Raupach and Shaw 1982; Finnigan 1985). Relatively recently attention has turned to the application of spatial averaging to open-channel flows (Nikora et al. 2001), for example gravelbed rivers and other channels with irregular rough surfaces. Flow subdivision into specific layers, based on spatial averaging methodology, is shown in Fig. 3 (Nikora et al. 2001). In this figure, the term roughness sublayer, used in atmospheric literature, is referred to as the roughness layer, as the roughness layer is itself subdivided into interfacial and form-induced sublayers lying immediately below and above the roughness tops, respectively (see Fig. 3). The term roughness layer has been adopted in this paper.

In spatial-averaging methodology, the Navier-Stokes equations are first time-averaged in the conventional way to produce the Reynolds averaged Navier-Stokes (RANS) equations. This is then supplemented by averaging over a volume or plane large enough to eliminate local variation in the mean flow due to bed topography (just as the temporal averaging time scale is chosen to eliminate the effect of turbulent fluctuations on the mean velocity). Supplementing Reynolds averaging with spatial averaging yields extra, physically meaningful terms including form drag and form-induced stress. Further details can be found in e.g. Raupach and Shaw (1982), Gimenez-Curto and Corniero Lera (1996), or Nikora et al. (2001). However, for convenience, the spatially averaged form of the Reynolds-averaged momentum equation (from Nikora et al. 2001) is presented here

$$
\begin{aligned}
\frac{\partial\left\langle\bar{u}_{i}\right\rangle}{\partial t}+\left\langle\bar{u}_{i}\right\rangle \frac{\partial\left\langle\bar{u}_{j}\right\rangle}{\partial x_{i}}= & g_{i}-\frac{1}{\rho} \frac{\partial\langle\bar{p}\rangle}{\partial x_{i}}+v \frac{\partial^{2}\left\langle\bar{u}_{i}\right\rangle}{\partial x_{j} \partial x_{j}}-\frac{1}{A} \frac{\partial A\left\langle\overline{u_{i}^{\prime} u_{j}^{\prime}}\right\rangle}{\partial x_{j}} \\
& -\frac{1}{A} \frac{\partial A\left\langle\tilde{u}_{i} \tilde{u}_{j}\right\rangle}{\partial x_{j}}+\nu\left\langle\frac{\partial^{2} \tilde{u}_{i}}{\partial x_{j} \partial x_{j}}\right\rangle-\frac{1}{\rho}\left\langle\frac{\partial \widetilde{p}}{\partial x_{i}}\right\rangle
\end{aligned}
$$

The straight overbar and angle brackets denote the time and spatial average of flow variables, respectively. The wavy overbar denotes the disturbance in the flow variables, i.e., the difference between time averaged $(\bar{V})$ and double averaged $(\langle\bar{V}\rangle)$ values $(\tilde{V}$ $=\bar{V}-\langle\bar{V}\rangle$, where $V=$ any flow variable defined in the fluid but not at points occupied by roughness elements); $g_{i}=$ gravity acceleration; $\rho=$ fluid density; $\nu=$ kinematic viscosity, and the roughness geometry function; and $A=$ ratio of the area (volume) occupied by fluid $A_{f}$ to the total averaging area (volume), $A_{0}$. Tensor notation is used for velocity subscripts.

Apart from the inclusion of the roughness geometry function $A$ and temporally averaged terms being replaced by their double averaged counterparts, the expressions in Eq. (1) are recognizable as those from the conventional RANS equations. The obvious exceptions are the last three terms. Taken in turn from left to right, these three terms represent the form-induced momentum flux, viscous drag, and form drag terms. The Reynolds stresses $\left(-\rho \overline{u_{i}^{\prime} u_{j}^{\prime}}\right)$ arise by substitution of mean $\left(\bar{u}_{i}\right)$ and fluctuating $\left(u_{i}^{\prime}\right)$ velocity components (i.e., $u_{i}=\bar{u}_{i}+u_{i}^{\prime}$ ) into the Navier-Stokes equation to obtain the Reynolds-averaged momentum equation (RANS). Form-induced stresses $\left(-\rho\left\langle\widetilde{u}_{i} \tilde{u}_{j}\right\rangle\right)$ appear following substitution into the RANS equation and subsequent averaging of a spatial mean velocity $\left(\left\langle\bar{u}_{i}\right\rangle\right)$ and its spatial "disturbance" $\left(\tilde{u}_{i}\right)$, i.e., $\bar{u}_{i}=\left\langle\bar{u}_{i}\right\rangle+\tilde{u}_{i}$. Hence the total fluid stress $\tau$, has three components: viscous, turbulent, and form-induced (or dispersive) stresses. For two-dimensional flow the total stress can be written as

$$
\tau=\rho\left\{\frac{v}{A} \frac{d(A\langle\bar{u}\rangle)}{d z}-\left\langle\overline{u^{\prime} w^{\prime}}\right\rangle-\langle\tilde{u} \widetilde{w}\rangle\right\}
$$

The PIV vector map data allow evaluation of spatial averages of flow variables in addition to temporal averages. Many results presented in the next section were therefore determined by a spatial averaging procedure. This process involved initial temporal averaging across the entire time series $(4,200$ frames at $16.7 \mathrm{~Hz})$ before spatial averaging along lines of constant distance from the mean bed in each vector field. The spatial averaging area was chosen to be representative of the fixed sediment bed topography throughout the flume.

\section{Results}

\section{Reynolds Shear Stresses}

Verification of two-dimensional (2D) flow conditions by demonstrating the linearity of the Reynolds stress distribution provides a logical starting point for presenting results. If adequately linear, the turbulent shear stress distributions, $\tau_{t}$, also offer an appropriate tool for evaluating bed shear stress $\tau_{0}$ and subsequently the shear velocity $u_{*}$ (Nezu and Nakagawa 1993). In this investigation the primary turbulent shear stress $\tau_{t}$ at level $z$ was determined from

$$
\left.\tau_{t}(z)=-\rho \overline{\left\langle u^{\prime} w^{\prime}\right.}\right\rangle
$$

where $u^{\prime}$ and $w^{\prime}$ are instantaneous fluctuations from the temporal mean longitudinal and vertical velocities, and averaging notation is as outlined for Eq. (1). Linear extrapolation of the Reynolds stress profile above the roughness layer (i.e., $z \geqslant 5 d$, where $d=d_{50}$, Raupach et al. 1991) to the mean bed level provided an estimate of the bed shear stress $\tau_{0}$, which was then used to evaluate shear velocity from

$$
u_{*}=\sqrt{\frac{\tau_{0}}{\rho}}
$$

Such values for the shear velocity (Table 1) were used for all normalizations of turbulence characteristics. Fig. 4 shows the turbulent shear stress distributions for all flow cases, illustrating that 

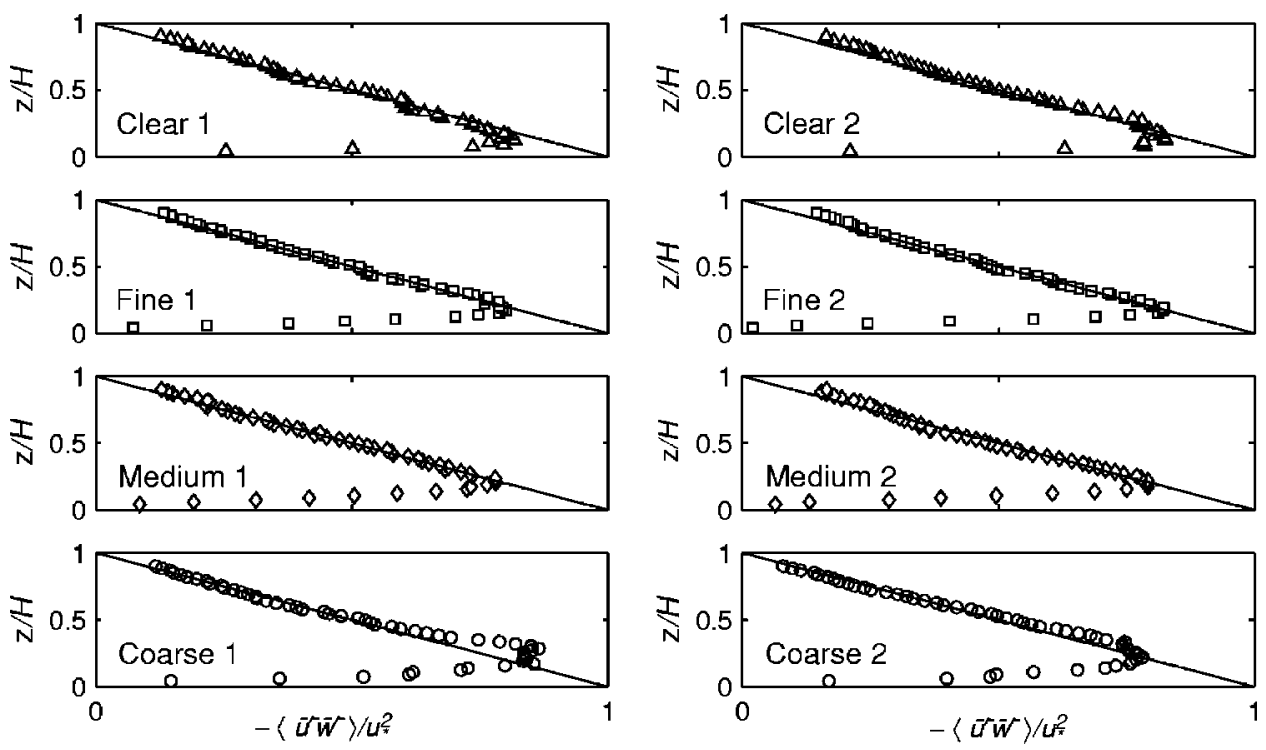

Fig. 4. Normalized Reynolds stress profiles for all eight experiments. Straight lines show ideal fit to data points.

$\tau_{t}=0$ at $z=H$, and $\tau_{t}=\tau_{0}=-\rho\left\langle\overline{u^{\prime} w^{\prime}}\right\rangle$ at the mean bed level.

The linear Reynolds stress trends in Fig. 4 demonstrate that the assumption of $2 \mathrm{D}$, uniform flow conditions is a valid one for the central part of our flume, where $-\overline{u^{\prime} w^{\prime}}$ is the dominant momentum flux. This was expected as the aspect ratio (flume width/flow depth, $B / H$ ) is close to 5 (Song and Graf 1994; Graf 1998). The primary shear stress deviates from linear in the near-bed region, indicating the presence of a roughness layer. This layer exists because the boundary imparts several momentum transformation mechanisms such as form drag, form-induced stresses, and when bed-load conditions prevail, an interaction between the flow and moving sediment. Fig. 4 indicates that the thickness of the roughness layer increased as the flow carried coarser material. The shear stress, $-\rho\left\langle\overline{u^{\prime} w^{\prime}}\right\rangle$ reached a maximum at $z \approx 6 \mathrm{~mm}$ for the clear water cases, but the corresponding heights for the fine, medium, and coarse feeds were $z \approx 10 \mathrm{~mm}, z \approx 13 \mathrm{~mm}$, and $z \approx 16 \mathrm{~mm}$, respectively. This trend of increasing roughness layer thickness with increasing bed-load feed size appears to be independent of sediment transport rate.

\section{Mean Velocities and Turbulence Intensities}

Figs. 5 and 6 show vertical distributions of double-averaged longitudinal velocity plotted semi-logarithmically with the vertical coordinate, $z$, normalized with mean flow depth $H$. Fig. 5 illustrates the influence of varying sediment size, whereas Fig. 6 indicates the relative importance of feed rate. Fig. 5(a) (lower feed rate, $0.003 \mathrm{~kg} / \mathrm{m} / \mathrm{s}$ ) reveals that for $z / H \leqslant 0.3$ the shift in velocity profiles is directly related to the sediment feed size, with the coarsest bed-load material causing the greatest retardation of longitudinal velocity. This effect has been observed to occur in sediment-laden flows and represents an increase in roughness (e.g., Best et al. 1997). Whether this is due to transient changes in bed roughness, or to dynamic roughness effects induced by moving sediment remains unclear. It is however worth noting that the coarse feed material had a tendency to become temporarily lodged on the fixed bed thereby intermittently forming transient clusters. Hence, the velocity profile shift for this case was at least partly attributable to increased (albeit temporarily) bed roughness caused by the clusters. Towards the free surface the coarse and fine materials similarly decelerate the longitudinal velocity, but the velocity above mobile medium sized sediment is indistinguishable from the clear water case.

Fig. 5(b) (higher feed rate, $0.006 \mathrm{~kg} / \mathrm{m} / \mathrm{s}$ ) shows that the mean flow profiles with coarse and medium sized bed-load particles are similar to the lower feed rate, but somewhat amplified. The coarse sediment has a prominent influence on the mean velocity throughout the flow depth whereas the profile shift caused by feeding medium sediment is again restricted to the near-bed region. The primary difference between the two feed rates is the flow behavior when fine sediment is transported. Feeding fine sediment at the lower feed rate $(0.003 \mathrm{~kg} / \mathrm{m} / \mathrm{s})$ results in lower downstream mean velocities throughout the flow depth as compared to the clear water case. However, when the feed rate of fine bed-load is doubled to $0.006 \mathrm{~kg} / \mathrm{m} / \mathrm{s}$, this velocity shift is reversed and mean longitudinal velocities are consistently higher than the clear water case. This effect is discussed further with reference to Figs. 6(a-d).

Fig. 6(a) illustrates the repeatability of the PIV data acquisition process, as the clear water velocity profiles obtained from Trials 1
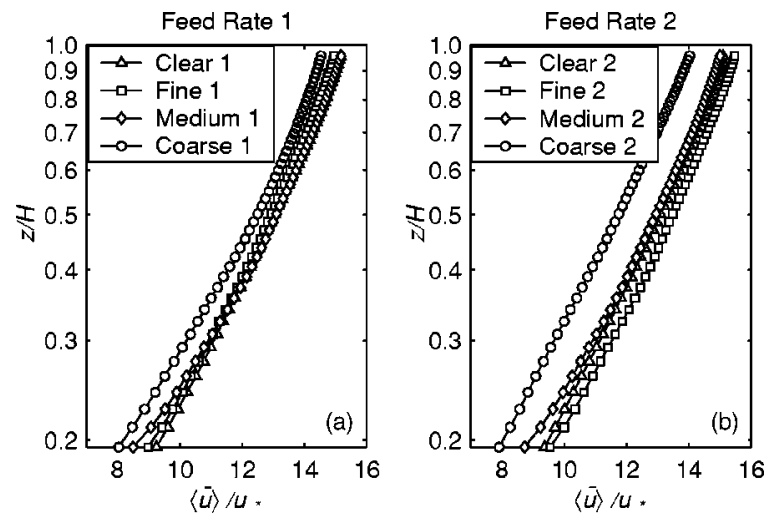

Fig. 5. Effect of bed-load particle size on double-averaged longitudinal velocity profiles. Data are normalized with depth and shear velocity, respectively: (a) lower feed rate $=0.003 \mathrm{~kg} / \mathrm{m} / \mathrm{s}$ and (b) higher feed rate $=0.006 \mathrm{~kg} / \mathrm{m} / \mathrm{s}$. 

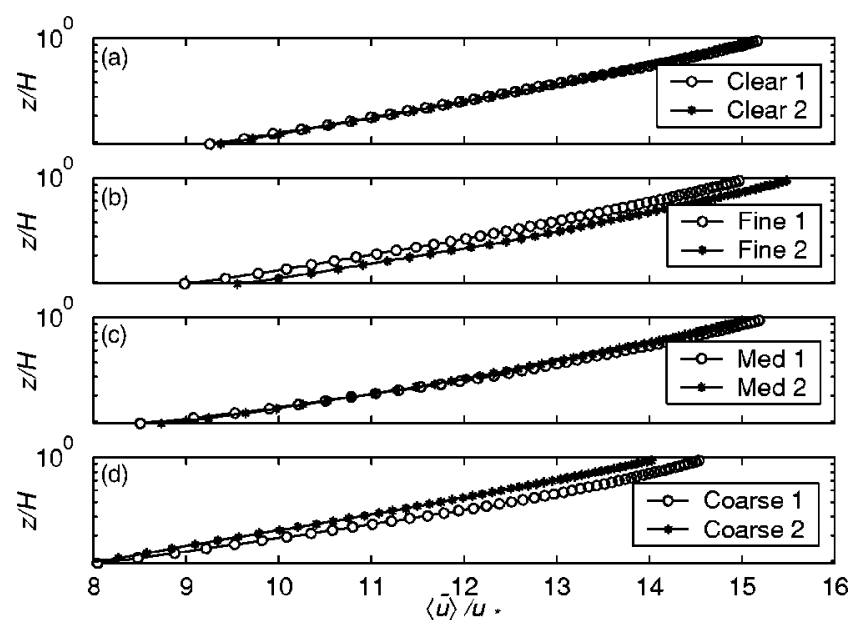

Fig. 6. Effect of feed rate on double-averaged longitudinal velocity profiles (normalized as in Fig. 5): (a) clear water (fixed bed $d_{50}$ $=1.95 \mathrm{~mm}$ ), (b) fine particles $\left(d_{50}=0.77 \mathrm{~mm}\right)$, (c) medium particles $\left(d_{50}=1.99 \mathrm{~mm}\right)$, and $(\mathrm{d})$ coarse particles $\left(d_{50}=3.96 \mathrm{~mm}\right)$; feed rate $1=0.003 \mathrm{~kg} / \mathrm{m} / \mathrm{s}$, feed rate $2=0.006 \mathrm{~kg} / \mathrm{m} / \mathrm{s}$

and 2 are essentially identical. The effect of increasing feed rate with the medium-sized bed-load material is negligible [Fig. 6(c)]. This is not true for either the fine or coarse sediment. Fig. 6(b) shows that feeding fine material at the higher rate reverses the initial shift in velocity profile observed with the lower feed rate, as noted when discussing Fig. 5(b). As the fine feed material $\left(d_{50}=0.77 \mathrm{~mm}\right)$ was transported through, and collected in the interstices of the bed surface, this effect was probably due to a decrease in the effective roughness of the bed. It is possible that such bed smoothing could only be fully realized when surplus bed-load material was made available at the higher feed rate and when the size distributions of the bed and mobile sediment permitted free exchange of the rolling bed-load grains with the interstices of the fixed bed.

Whiting and Dietrich (1990) concluded that flow resistance with bed load was indistinguishable from clear water flows. They suggested that the coarse bed-load fraction moves by rolling slowly over the bed compared to the fluid velocity, and that momentum dissipation by the drag of the bed occurs in the same manner as over immobile beds. Furthermore, the fine bed-load fraction moves below the tops of the large grains and grains clusters and has negligible effect on flow resistance. Whereas Whiting and Dietrich (1990) had the same sediment compositions for the bed and bed-load grains, this study introduces bed-load size fractions much smaller and larger than the fixed bed sediment. Figs. 5 and 6 show that both bed-load size and transport rate exert a more complex influence on the mean flow velocity than that proposed by Whiting and Dietrich (1990). However, their observation that flow resistance is indistinguishable for bed load and clear water flows is supported by our experiments involving the bed-load transport of medium grains. In this case, where the fixed bed and bed-load size fractions are similar (Fig. 1) the mean flow is relatively unaffected compared to the clear water condition.

As there is no strong physical justification for the existence of a well-developed logarithmic layer with the combinations of flow depth and roughness heights in this study, no log law parameters were calculated from the velocity profile. For example, in the clear water cases the characteristic roughness height $\left(d=d_{50}\right)$ was $1.95 \mathrm{~mm}$, and the flow depth $(H)$ was $66 \mathrm{~mm}$. Hence we would expect the overlap between the inner and outer layers to lie in the
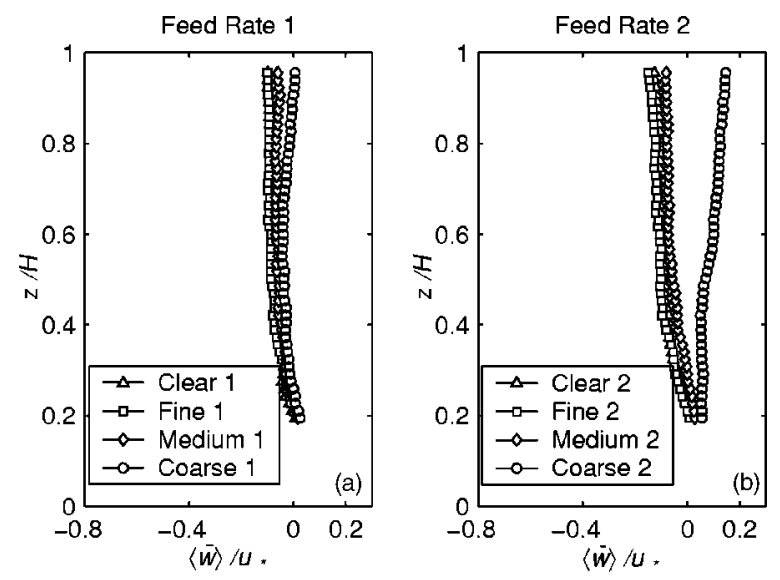

Fig. 7. Double-averaged bed-normal velocity profiles (normalized as in Fig. 5) for: (a) feed rate $1(0.003 \mathrm{~kg} / \mathrm{m} / \mathrm{s})$ and (b) feed rate 2 $(0.006 \mathrm{~kg} / \mathrm{m} / \mathrm{s})$

range $9.75 \leqslant z \leqslant 13.2 \mathrm{~mm}[5 d \leqslant z \leqslant 0.2 H$, Raupach et al. (1991), alternatively Jimenez (2004) recommends the upper bound of logarithmic behavior lies at $z=0.15 H$ ]. This range gives just under $3.5 \mathrm{~mm}$ of justified logarithmic behavior, containing only three data points. For the sediment feed cases, the logarithmic layer diminishes as it becomes squeezed between the thickening roughness layer and the outer layer, and it would therefore be misleading to assess variables (e.g., shear velocity, roughness lengths, eddy viscosity) based on the log law.

In all experiments the double averaged vertical velocity remained close to zero (Fig. 7), suggesting that there was no consistent organized vertical momentum flux. This provides further support for the lack of interference from secondary circulation in the flume midline.

Figs. 8(a-d) illustrate the relative longitudinal and vertical turbulence intensities, $\sigma_{i}=\sqrt{\left\langle\overline{u_{i}^{\prime} u_{i}^{\prime}}\right\rangle} / u_{*}$, for feed rates 1 and 2 . For both feed rates longitudinal turbulence intensity increased near the bed up to around $z / H=0.4$ during feeding of all particle sizes [Figs. 8(a and b)]. All grain sizes caused increased values of vertical turbulence intensity $\sigma_{w} / u_{*}$ above $z / H=0.25$, while nearbed values of $\sigma_{w} / u_{*}$ were decreased, compared to the clear water case. Enhanced longitudinal turbulence intensity throughout much of the flow depth in the presence of bed load agrees with previous research (Wang and Larsen 1994; Best et al. 1997; Song and Chiew 1997) and supports the idea that bed load extracts momentum from the mean flow. Between $z / H=0.2$ and 0.5 the vertical turbulence intensity with the higher feed rate of coarse grains [Fig. 8(d)] is increased by up to $20 \%$ compared to the clear water case. This is another indication that the "stati" bed roughness was enhanced with the transport of coarse bed load.

\section{Turbulent Kinetic Energy Production}

In the vicinity of the roughness tops there is a zone of high velocity gradient (Fig. 9) and peak turbulent kinetic energy (TKE) production (Fig. 10). Turbulent "shear" production $\left(P_{s}\right)$ is the product of the primary turbulent momentum flux and the mean longitudinal velocity gradient. In terms of double-averaged variables, this is 
Feed Rate 1
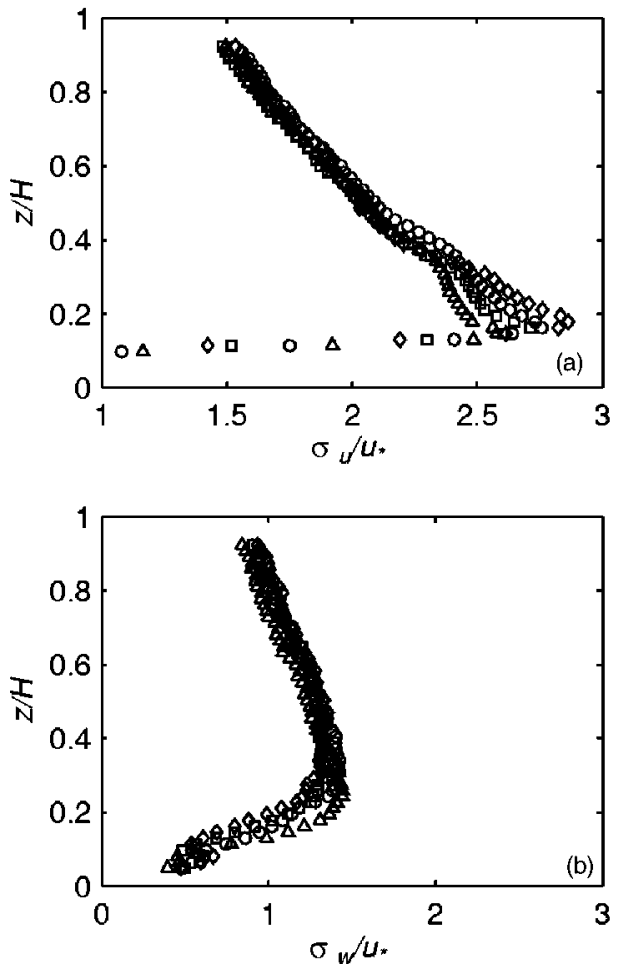

Feed Rate 2
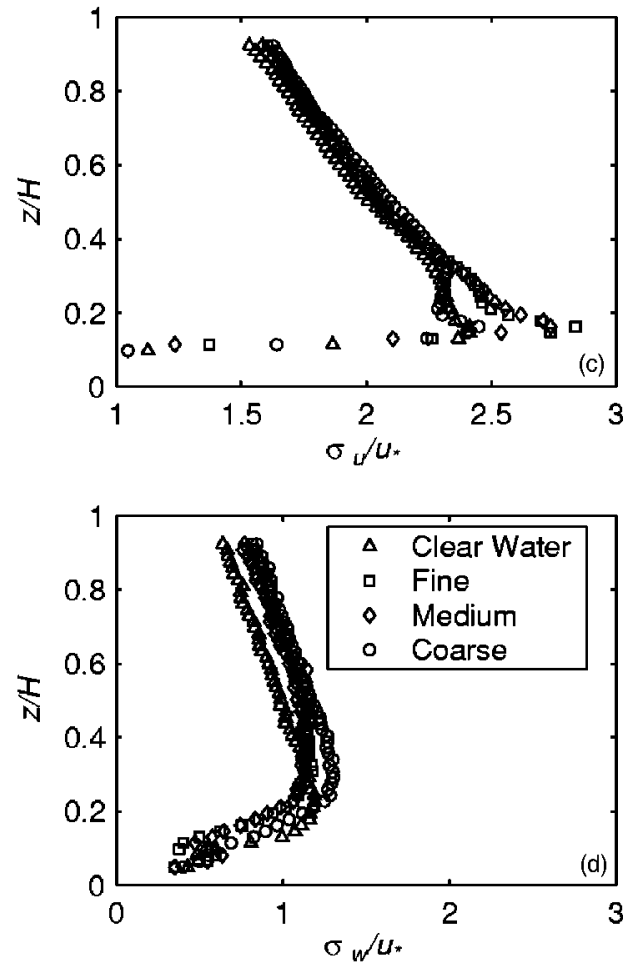

Fig. 8. Turbulence intensities (root mean square of velocity fluctuations) normalized with shear velocity: (a) longitudinal turbulence intensity, feed rate $1(0.003 \mathrm{~kg} / \mathrm{m} / \mathrm{s})$, (b) vertical turbulence intensity, feed rate 1 , (c) longitudinal turbulence intensity, feed rate $2(0.006 \mathrm{~kg} / \mathrm{m} / \mathrm{s})$, and (d) vertical turbulence intensity, feed rate 2 . In all cases standard errors in measurements are comparable to, or smaller than, symbol dimensions.

$$
\left.P_{s}=-\overline{\left\langle u^{\prime} w^{\prime}\right.}\right\rangle \frac{\partial\langle\bar{u}\rangle}{\partial z}
$$

Full evaluation of the TKE production requires knowledge of additional terms describing the wake production and the energy transfers due to fluid-sediment interactions. As these terms could not be evaluated it is not possible to draw conclusions about potential differences in overall energy production and dissipation rates between clear water and bed-load cases.

The coarse sediment feed conditions produced the highest Reynolds stresses in the near-bed region, with little difference between the clear water, fine and medium feed cases. However,
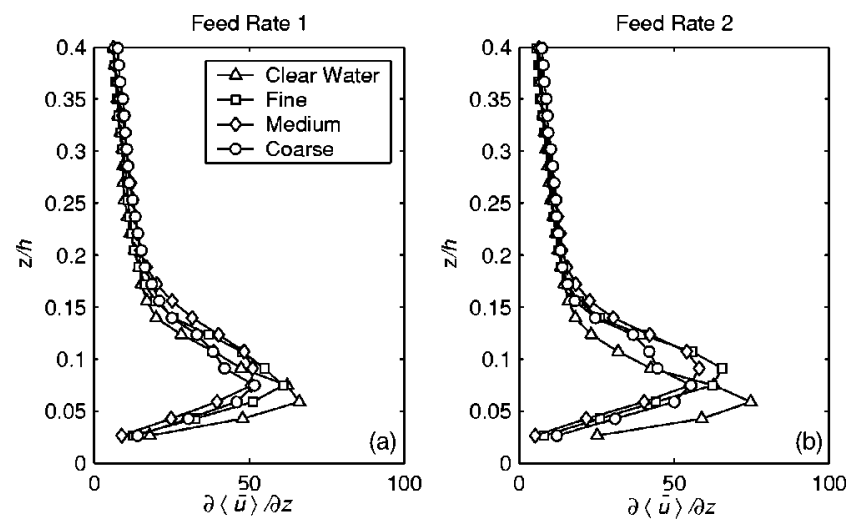

Fig. 9. Double-averaged velocity gradients, $\partial\langle\bar{u}\rangle / \partial z$, up to $z=0.4 H$ for: (a) feed rate $1(0.003 \mathrm{~kg} / \mathrm{m} / \mathrm{s})$, and (b) feed rate 2 $(0.006 \mathrm{~kg} / \mathrm{m} / \mathrm{s})$ the mean longitudinal velocity gradients apparently play the dominant role in determining shear production (Fig. 9). Previous researchers (e.g., Muller 1973) have noted an increase in velocity gradient with bed-load transport. Compared to the clear water control experiments, for all bed-load cases the streamwise velocity gradient was increased across the range $0.075 \leqslant z / H \leqslant 0.4$. However, the magnitude of the increase in streamwise velocity gradients in the presence of bed load does not appear to be related to sediment feed size in any organized manner. Although the clear water experiments have the maximum velocity gradient below
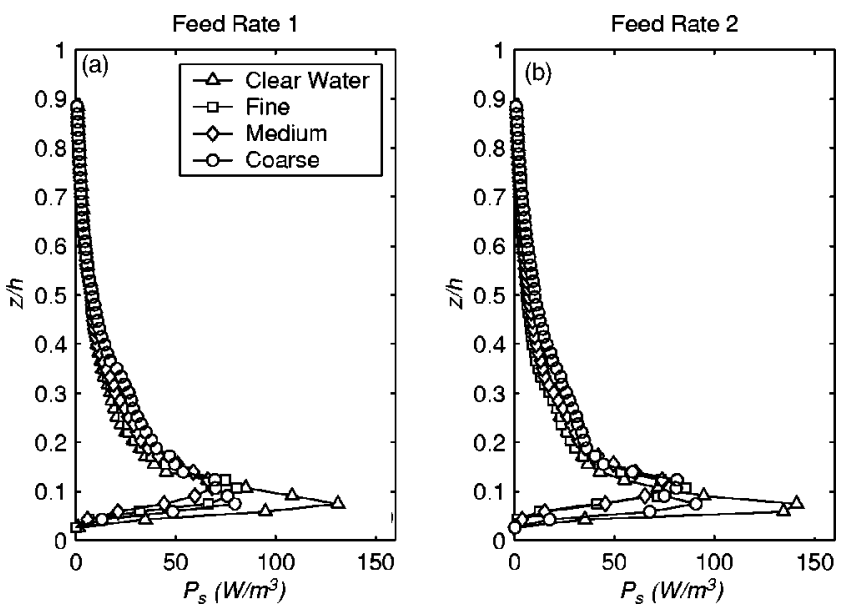

Fig. 10. Double-averaged turbulent kinetic energy shear production $P_{s}$ for: (a) feed rate $1(0.003 \mathrm{~kg} / \mathrm{m} / \mathrm{s})$ and (b) feed rate 2 $(0.006 \mathrm{~kg} / \mathrm{m} / \mathrm{s})$ 

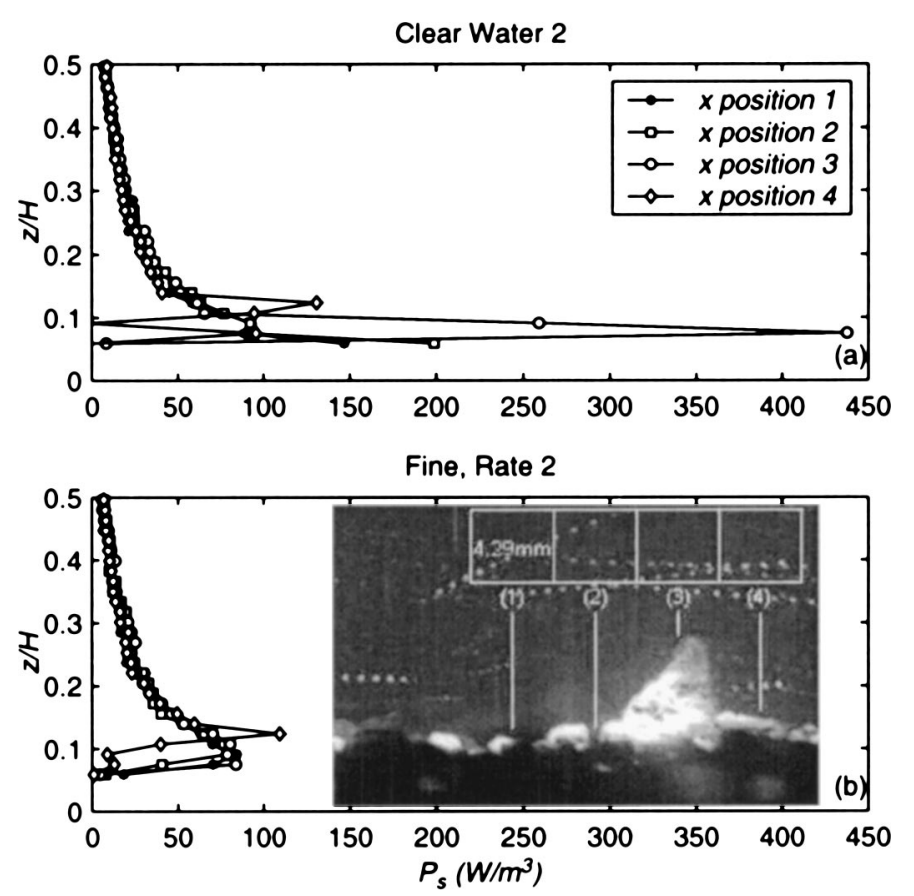

Fig. 11. Local turbulent kinetic energy production for four verticals over fixed downstream particle cluster for $z / H<0.5$ : (a) clear water 2 and (b) fine bed-load particles at feed rate $2(0.006 \mathrm{~kg} / \mathrm{m} / \mathrm{s})$. Image inset comes from experiment "Clear Water 1." Also shown are relevant $4.29 \mathrm{~mm} \times 4.29 \mathrm{~mm}$ particle image velocimetry interrogation areas. Data for above graphs come from nonoverlapped regions.

$z / H \sim 0.075$, the results from this area of the flow must be treated with caution due to potential interference from mobile sediment in all bed-load cases. The spatially averaged TKE shear production profiles (Fig. 10) reveal suppressed turbulent energy generation very close to the bed for all transporting cases. Above $z / H$ $=0.1$, flows carrying bed load generally exhibited higher turbulent shear production compared to the clear water cases, with the degree of increase being dependent on bed-load particle size (largest particles causing the greatest increase). The TKE production for the experiments "coarse 1" and "coarse 2" was consistently greater than the clear water value through much of the flow depth. This was also true for experiments "medium 1" and "medium 2," but the effect was only discernible up to $z / H \approx 0.3$. Although for $z / H>0.15$ the fine bed-load particles increased the TKE shear production compared to the clear water case at feed rate 1 $(0.003 \mathrm{~kg} / \mathrm{m} / \mathrm{s})$, slightly decreased levels were observed for feed rate $2(0.006 \mathrm{~kg} / \mathrm{m} / \mathrm{s})$. This again can be attributed to some degree of bed smoothing.

The PIV data collection also provided the opportunity to investigate spatial variation in TKE production. This made it possible to examine the bed topography to identify potential concentrations in TKE production. The fixed downstream particle cluster evident in Fig. 2(b) was therefore examined. Fig. 11(a) shows local TKE (i.e., without spatial averaging) production profiles for four verticals situated at various positions over the cluster for the clear water case, and Fig. 11(b) shows the corresponding profiles for the high feed-rate fine particles. These plots clearly demonstrate both the existence of a local concentration of TKE generation and the leveling influence of the fine bed-load particles as
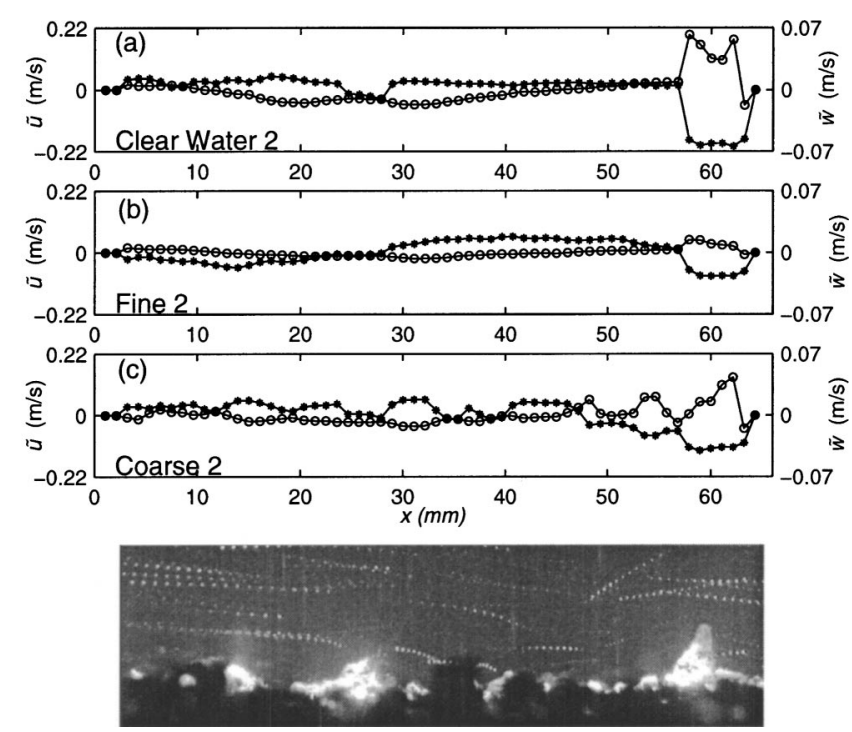

Fig. 12. Form-induced velocity components, $\tilde{u}(*)$ and $\tilde{w}(\bigcirc)$ at $z=6.03 \mathrm{~mm}$ above mean bed level, $z / H=0.091$ (note different scales used for plotting $\widetilde{u}$ and $\widetilde{w}$ ). Particle image velocimetry image shows underlying fixed bed topography for clear water case: (a) no bed load; (b) fine bed-load particles at high feed rate $(0.006 \mathrm{~kg} / \mathrm{m} / \mathrm{s})$; and (c) coarse bed load at high feed rate $(0.006 \mathrm{~kg} / \mathrm{m} / \mathrm{s})$.

they smooth the bed surface, reducing the peak TKE production to $15 \%$ of the clear water case. These observations highlight an issue of intermittency in the turbulent energy production that may appear to be a key issue for understanding various near-bed transport processes.

\section{Form-Induced Stresses}

Further evidence for bed smoothing can be found by examining the difference between time-averaged and double-averaged flow variables. The variation in $\tilde{u}$ and $\tilde{w}$ along the flow is presented in Figs. 12(a-c). With no bed-load transport, the spatial disturbance around the downstream particle cluster is characterized by
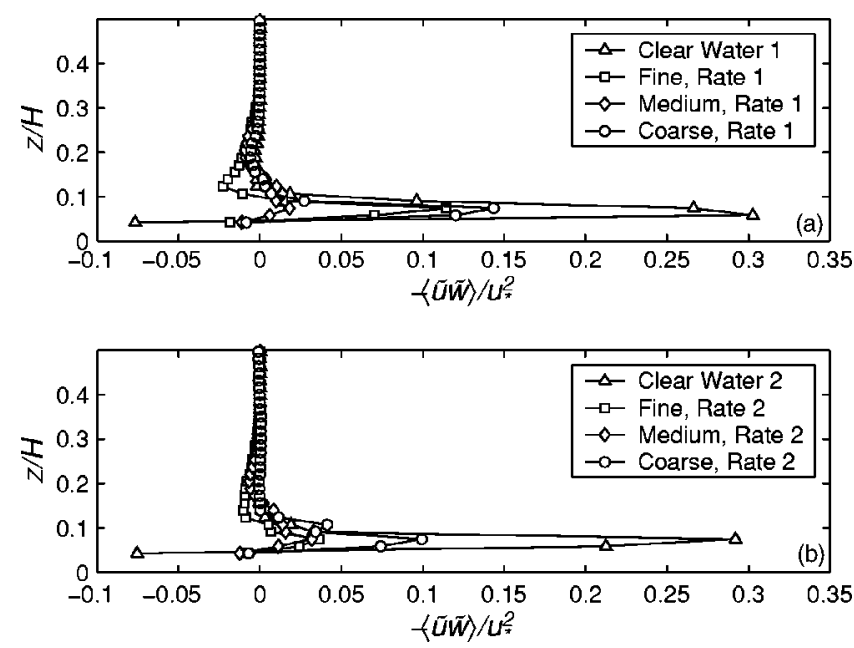

Fig. 13. Normalized form-induced stress profiles $-\rho\langle\tilde{u} \tilde{w}\rangle / u_{*}^{2}$ for: (a) feed rate $1(0.003 \mathrm{~kg} / \mathrm{m} / \mathrm{s})$ and (b) feed rate $2(0.006 \mathrm{~kg} / \mathrm{m} / \mathrm{s})$ 
a large retardation in streamwise velocity, accompanied by an augmented vertical velocity as flow is forced over and around the grains [Fig. 12(a)]. This effect was greatly reduced in the "fine 2" case, indicating that the bed irregularities had been smoothed to some extent [Fig. 12(b)]. In contrast, the overall spatial fluctuations $(\widetilde{u}$ and $\widetilde{w})$ associated with coarse bed-load particles were far more erratic as the bed roughness was greatly enhanced [Fig. 12(c)]. Studies that have considered spatial averaging of flow variables thus far (primarily within plant canopies) have proposed that the maximum form-induced stress would be, at most, only a few percent of the Reynolds stress in the vicinity of the bed roughness (Mulhearn 1978; Raupach and Thom 1981; Raupach et al. 1986; Perry et al. 1987). Fig. 13 shows that for the fixed bed, the form-induced stress approached $30 \%$ of the maximum Reynolds stress. Although form-induced stress levels were reduced with mobile sediment they still constituted up to $15 \%$ of the total measured fluid stress (turbulent+form induced) in the roughness layer.

\section{Conclusions}

This study provides new information about how turbulent flow structure is affected by the size and feed rate of bed load. Velocities were measured using PIV that provided instantaneous, timeaveraged, and double-averaged hydrodynamic fields in clearwater and bed-load flows. The results of this investigation supplement and support previous findings and may be summarized as follows:

1. With increase in feeding rate, the downwards shift in mean longitudinal velocity profile increased for the coarse case, remained constant for the medium case, and reversed for the fine case (i.e., velocities in the "fine 2" experiment were higher than observed for the clear water case).

2. The TKE shear production increased for all bed-load cases above $z / H=0.1$. The largest increase above the roughness layer was observed for the coarse feed condition. The gradient of streamwise velocity increased for all bed-load cases across the region $0.075 \leqslant z / H \leqslant 0.4$.

3. Local concentrations in TKE production were smoothed in the fine feed case but little change was observed for the medium and coarse experiments.

4. Form-induced stress levels remained relatively constant for both the medium and coarse bed particles. Increasing the feed rate for the fine particles caused the average forminduced stress to drop by over $50 \%$.

5. For all bed-load cases the form-induced stress is decreased compared to the clear water experiments. Nevertheless, form-induced stress contributes up to $15 \%$ of the maximum Reynolds stress in the roughness layer in the presence of bed load.

6. The recorded values of $\sigma_{u} / u_{*}$ and $\sigma_{w} / u_{*}$ increased in all cases for $z / H>0.25$. Closer to the bed, $\sigma_{w} / u_{*}$ decreased for the coarse case and increased for the fine and medium cases, compared to the clear-water values.

As a final note, it is important to emphasize that these results were obtained over a nonporous planar boundary. It is most likely that differences in momentum transfer will occur over natural, porous sediment beds where particles are free to move. Further work is required over such beds to develop better understanding of flow modification due to bed-load transport.

\section{Acknowledgments}

This investigation was carried out as part of an experimental program funded by U.K. EPSRC (Grant No. GR/L54448/01). The study was also supported by the Marsden Fund administered by the Royal Society of New Zealand (Contract No. NIW001).

\section{Notation}

The following symbols are used in this paper:

$A=$ roughness geometry function, ratio of area $A_{f}$ occupied by fluid to fixed averaging region $A_{o}$ in $x, y$ plane;

$B=$ flume width;

$d_{50}=$ grain size, where subscript denotes "percentage finer than";

$\mathrm{F}=$ Froude number;

$g=$ gravity acceleration;

$H=$ mean flow depth;

$P_{s}=$ turbulent kinetic energy shear production

$R=$ hydraulic radius;

$\mathrm{R}=$ Reynolds number;

$\mathrm{R}_{*}=$ roughness Reynolds number;

$\bar{U}=$ depth averaged velocity;

$u_{*}=$ shear (friction) velocity;

$\overline{u_{i}^{\prime} u_{j}^{\prime}}=$ local Reynolds (turbulent) stress;

$\left\langle\overline{u_{i}^{\prime} u_{j}^{\prime}}\right\rangle=$ double-averaged Reynolds (turbulent) stress;

$\left\langle\tilde{u}_{i} \tilde{u}_{j}\right\rangle=$ form-induced (or dispersive) stress;

$u, v, w=$ instantaneous longitudinal, transverse, and vertical velocity components, respectively;

$\bar{u}, \bar{v}, \bar{w}=$ time-averaged velocity components, i.e., $\bar{u}_{i}$ $=u_{i}-u_{i}^{\prime}$;

$\tilde{u}, \tilde{v}, \widetilde{w}=$ form-induced velocity components, i.e., $\tilde{u}_{i}=\bar{u}_{i}$ $-\left\langle\bar{u}_{i}\right\rangle$;

$u^{\prime}, v^{\prime}, w^{\prime}=$ flow velocity fluctuation components about temporal mean, i.e., $u_{i}^{\prime}=u_{i}-\bar{u}_{i}$;

$\langle\bar{u}\rangle,\langle\bar{v}\rangle,\langle\bar{w}\rangle=$ double-averaged (in time and spatial domains) velocity components, i.e., $\left\langle\bar{u}_{i}\right\rangle=\bar{u}_{i}+\widetilde{u}_{i}$;

$x, y, z=$ coordinate axes (longitudinal, transverse, and bed normal);

$\theta=$ Shield's parameter;

$v=$ kinematic viscosity;

$\rho=$ density of water;

$\sigma_{i}=$ root mean square of velocity fluctuations (turbulence intensities);

$\tau=$ total fluid stress;

$\tau_{t}=$ Reynolds (turbulent) stress; and

$\tau_{0}=$ bed shear stress.

\section{References}

Best, J., Bennett, S., Bridge, J., and Leeder, M. (1997). "Turbulence modulation and particle velocities over flat sand beds at low transport rates." J. Hydraul. Eng., 123(12), 1118-1129.

Carbonneau, P. E., and Bergeron, N. E. (2000). "The effect of bedload transport on mean and turbulent flow properties." Geomorphology, $35,267-278$.

Finnigan, J. J. (1985). "Turbulent transport in flexible plant canopies." The forest-atmosphere interactions, B. A. Hutchinson and B. B. Hicks, eds., Reidel, Dordrecht, The Netherlands, 443-480.

Gimenez-Curto, L. A., and Corniero Lera, M. A. (1996). "Oscillating 
turbulent flow over very rough surfaces." J. Geophys. Res., 101(C9), 20745-20758.

Gore, R. A., and Crowe, C. T. (1989). "Effect of particle size on modulating turbulent intensity." Int. J. Multiphase Flow, 15, 279-285.

Graf, W. H. (1998). "Fluvial hydraulics: Flow and transport processes in channels of simple geometry," Wiley, Chichester, U.K.

Gust, G., and Southard, J. B. (1983). "Effects of weak bed load on the universal law of the wall." J. Geophys. Res., 88(C10), 5939-5952.

Gyr, A., and Schmid, A. (1997). "Turbulent flows over smooth erodible sand beds in flumes." J. Hydraul. Res., 35(4), 525-544.

Jimenez, J. (2004). "Turbulent flows over rough walls." Annu. Rev. Fluid Mech., 36, 173-196.

Mulhearn, P. J. (1978). "Turbulent flow over a periodic rough surface." Phys. Fluids, 21, 1113-1115.

Müller, A. (1973). "Turbulence measurements over a movable bed with sediment transport by laser anemometry." Proc., 15th Congress, International Association of Hydraulic Research, Vol. 1, A7-1-A7-7.

Nezu, I., and Nakagawa, H. (1993). Turbulence in open-channel flows, Balkema, Rotterdam, The Netherlands.

Nikora, V., and Goring, D. (2000). "Flow turbulence over fixed and weakly mobile gravel beds." J. Hydraul. Eng., 126(9), 679-690.

Nikora, V., Goring, D., McEwan, I., and Griffiths, G. (2001). "Spatiallyaveraged open-channel flow over rough bed." J. Hydraul. Eng., 127(2), 123-133.

Owen, P. R. (1964). "Saltation of uniform grains in air." J. Fluid Mech., 20, 225-242.

Perry, A. E., Lim, K. L., and Henbest, S. M. (1987). "An experimental study of turbulence structure in smooth- and rough-wall turbulent boundary layers." J. Fluid Mech., 177, 437-466.

Pitlick, J. (1992). "Flow resistance under conditions of intense gravel transport." Water Resour. Res., 28, 891-903.

Raupach, M. R., Antonia, R. A., and Rajagopalan, S. (1991). "Rough- wall turbulent boundary layers." Appl. Mech. Rev., 44(1), 1-24.

Raupach, M. R., Coppin, P. A., and Legg, B. J. (1986). "Experiments on scalar dispersion within a plant canopy 1, the turbulence structure." Boundary-Layer Meteorol., 35, 21-52.

Raupach, M. R., and Shaw, R. H. (1982). "Averaging procedures for flow within vegetation canopies." Boundary-Layer Meteorol., 22, 79-90.

Raupach, M. R., and Thom, A. S. (1981). "Turbulence in and above plant canopies." Annu. Rev. Fluid Mech., 13, 97-129.

Shand, A. M. (1996). "The investigation, development and optimisation of global laser diagnostics for combustion and related flow applications." PhD thesis, Univ. of Warwick.

Smith, J. D., and McLean, S. R. (1977). "Spatially averaged flow over a wavy surface." J. Geophys. Res., 82(12), 1735-1746.

Song, T., and Chiew, Y. M. (1997). "Effect of bedload movement on flow resistance." Proc., 27th IAHR Congress: Environmental and Coastal Hydraulics, San Francisco.

Song, T., and Graf, W. H. (1994). "Non-uniform open-channel flow over a rough bed." J. Hydrosci. Hydr. Eng., 12(1), 1-25.

Vanoni, V. A., and Nomicos, G. N. (1960). "Resistance properties of sediment laden streams." Trans. Am. Soc. Civ. Eng., 125, 1140-1175.

Wang, X., and Ning, Q. (1989). "Turbulence characteristics of sediment laden flow." J. Hydraul. Eng., 115(6), 781-800.

Wang, Z., and Larsen, P. (1994). "Turbulent structure of water and clay suspensions with bed load." J. Hydraul. Eng., 120(5), 577-600.

Westerweel, J. (1994). "Efficient detection of spurious vectors in particle image velocimetry data." Exp. Fluids, 16, 236-247.

Whiting, P. J., and Dietrich, W. E. (1990). "Boundary shear stress and roughness over mobile alluvial beds." J. Hydraul. Eng., 116(12), $1495-1511$.

Wilson, N. R., and Shaw, R. H. (1977). "A higher order closure model for canopy flow." J. Appl. Meteorol., 16, 1197-1205. 